\title{
ELP3 Is Involved in Sexual and Asexual Development, Virulence, and the Oxidative Stress Response in Fusarium graminearum
}

\author{
Yoonji Lee, ${ }^{1}$ Kyunghun Min, ${ }^{1}$ Hokyoung Son, ${ }^{1}$ Ae Ran Park, ${ }^{1}$ Jin-Cheol Kim, ${ }^{2}$ Gyung Ja Choi, ${ }^{2}$ and \\ Yin-Won Lee ${ }^{1}$ \\ ${ }^{1}$ Department of Agricultural Biotechnology and Center for Fungal Pathogenesis, Seoul National University, Seoul 151-921, \\ Republic of Korea; ${ }^{2}$ Eco-friendly New Materials Research Group, Research Center for Biobased Chemistry, Division of \\ Convergence Chemistry, Korea Research Institute of Chemical Technology, Daejeon 305-343, Republic of Korea
}

Submitted 20 May 2014. Accepted 22 July 2014.

\begin{abstract}
Fusarium graminearum is an important fungal plant pathogen that causes serious losses in cereal crop yields and mycotoxicoses in humans and livestock. In this study, we characterized an insertion mutant, Z39R9282, with pleiotropic defects in sexual development and virulence. We determined that the insertion occurred in a gene encoding an ortholog of yeast elongator complex protein 3 (ELP3). Deletion of elp3 led to significant defects in sexual and asexual development in F. graminearum. In the elp3 deletion mutant, the number of perithecia formed was reduced and maturation of perithecia was delayed. This mutant also produced morphologically abnormal ascospores and conidia. Histone acetylation in the elp3 deletion mutant was reduced compared with the wild type, which likely caused the developmental defects. Trichothecenes were not produced at detectable levels, and expression of trichothecene biosynthesis genes were significantly reduced in the elp3 deletion mutant. Infection of wheat heads revealed that the elp3 deletion mutant was unable to spread from inoculated florets to neighboring spikelets. Furthermore, the elp3 deletion mutant was more sensitive to oxidative stress than the wild type, and the expression of putative catalase genes was reduced. We demonstrate that elp3 functions in sexual and asexual development, virulence, and the oxidative stress response of $F$. graminearum by regulating the expression of genes involved in these various developmental processes.
\end{abstract}

The homothallic ascomycete fungus Fusarium graminearum is an important plant pathogen of major cereal crops such as wheat, maize, barley, and rice (Leslie and Summerell 2006). Epidemics of Fusarium head blight (FHB) caused by F. graminearum result in serious losses in wheat and barley yields. Infected grains are commonly contaminated with mycotoxins such as trichothecenes and zearalenone that can lead to mycotoxicoses in humans and livestock (Desjardins and Proctor 2007).

The random insertion mutagenesis method called restriction enzyme-mediated integration (REMI) was first described in Saccharomyces cerevisiae (Schiestl and Petes 1991) and was

Corresponding author: Yin-Won Lee: E-mail: lee2443@snu.ac.kr

* The $e$-Xtra logo stands for "electronic extra" and indicates that six supplementary figures and one supplementary table are published online.

(C) 2014 The American Phytopathological Society later used to identify pathogenicity genes in several plantpathogenic fungi, including Cochliobolus heterostrophus, Ustilago maydis, and Magnaporthe grisea (Bölker et al. 1995; Lu et al. 1994; Sweigard et al. 1998). REMI has been used to identify genes involved in $F$. graminearum sexual development and virulence (Han et al. 2004; Kim et al. 2009; Seong et al. 2005; Wang et al. 2011).

For pathogenic fungi to successfully colonize their hosts, various transcriptional regulatory mechanisms must control gene expression in response to environmental perturbations such as osmotic and oxidative stress. Post-translational modifications of chromatin such as histone acetylation are closely associated with transcriptional activation (Wolffe and Pruss 1996). Acetylation of lysine residues within the $\mathrm{N}$-terminal tails of histone proteins changes chromatin structure by neutralizing positively charged nucleosomes and, consequently, increasing the accessibility of transcription factors to their target genes (Grunstein 1997). Histone acetylation is mediated by histone acetyltransferases (HAT) and is reversed by histone deacetylases (HDAC). HAT transfer the acetyl groups from acetyl-coenzyme A to the lysine residues of histones, whereas HDAC remove the acetyl groups (Kuo and Allis 1998). The general control nonderepressible 5 (GCN5)related acetyltransferase (GNAT) superfamily is one of the conserved HAT families (Carrozza et al. 2003), and members of the GNAT family have similar three-dimensional structures and catalytic domains (Vetting et al. 2005).

Elongator protein 3 (ELP3), a member of the GNAT family, is the catalytic subunit of the Elongator complex. Elongator complex was first identified in $S$. cerevisiae as a multisubunit complex associated with elongating RNA polymerase II (RNAPII) (Otero et al. 1999); it was later found to be composed of six subunits, ELP1 to ELP6. In yeast, purified ELP3 acetylated histones in vitro, and the HAT activity of ELP3 was essential for its function in vivo (Wittschieben et al. 1999, 2000). Mammalian ELP3 was also purified and its ability to acetylate histones $\mathrm{H} 3$ and $\mathrm{H} 4$ was verified (Hawkes et al. 2002).

Previous studies revealed that elp3 was required for the activation of a group of stress-inducible genes in eukaryotes (Creppe and Buschbeck 2011; Han et al. 2007a; Nelissen et al. 2010; Wittschieben et al. 2000). In S. cerevisiae, loss of elp3 reduced the activation of genes involved in the adaptation to stressful growth conditions (Wittschieben et al. 2000). In Arabidopsis, elp3 was required for the transcription of stressinduced and auxin-related genes (Chen et al. 2006; Nelissen et al. 2010). Using human cells, elp3 was found to regulate the heat shock response gene, $h s p 70$ (Han et al. 2007a). Although 
the biochemical function of elp3 is conserved in eukaryotes, elp3 target genes vary by species.

In this study, we identified the $F$. graminearum ortholog of $S$. cerevisiae elp3 using a forward genetics approach. The $F$. graminearum elp3 deletion mutant showed defects in sexual and asexual development, trichothecene production, virulence, and the oxidative stress response. We hypothesized that the pleiotropic defects seen in the elp 3 deletion mutant was caused by reduced histone acetylation and that elp3 might play a role in regulating pathogenicity genes in $F$. graminearum. The objectives of this study were to characterize the roles of elp3 in $F$. graminearum and to identify the stress-inducible genes regulated by elp3. Our results demonstrated that elp3 functioned in sexual and asexual development, virulence, and regulation of oxidative stress-induced genes in F. graminearum.

\section{RESULTS}

\section{Identification of elp3.}

A REMI mutant named Z39R9282 exhibited defects in sexual development and virulence relative to the wild-type strain (Fig. 1A). This mutant also had reduced aerial mycelia and excess accumulation of red pigments (Fig. 1A). Southern hybridization of Z39R9282 BglII-digested-genomic DNA using pBCATPH as a probe revealed that single insertion of the vector had occurred. Plasmid rescue was utilized to identify the insertion site, and it was found to be at the HindIII site located 274 bp downstream of the start codon of FGSG_02040.3, which encodes an ortholog of yeast ELP3 (Fig. 1B; Supplementary Fig. S1A). ELP3 contains a HAT domain, a putative S-adenosylmethionine-binding domain, and iron-sulfur cluster domains. ELP3 is highly conserved among eukaryotes, and a phylogenetic analysis of ELP3 orthologs revealed that they clustered into distinct groups according to their phyla. Fungal orthologs of ELP3 clustered into two groups corresponding to the subphyla Pezizomycotina and Saccharomycotina (Fig. 1C).

\section{Targeted deletion and complementation.}

To elucidate the functions of elp3 in F. graminearum, targeted gene deletion and complementation strains were created by homologous recombination. The elp3 open reading frame (ORF) in the $F$. graminearum wild-type strain Z-3639 was replaced with the geneticin resistance gene (gen) to create the elp 3 deletion mutant strain. To generate the elp 3 complementation strain, gen was replaced on the wild-type allele of elp3 fused to the hygromycin resistance gene (hyg). Southern hybridizations were performed on SalI-digested genomic DNA from these strains using the $5^{\prime}$ flanking region of elp3 as a probe, and these hybridizations confirmed successful gene deletion and complementation (Supplementary Fig. S2). When cultured on complete media (CM), the elp3 deletion mutant produced sparse aerial mycelia and more red pigments compared with the wild-type and complementation strains (Fig. 2A). However, there was no significant difference in radial growth among the strains (Table 1). The elp3 deletion mutant showed indistinguishable phenotypes with an REMI mutant Z39R9282 in sexual and asexual development, virulence, and toxin production (data not shown).

\section{Sexual and asexual development.}

When self-fertilized, the elp3 deletion mutant produced fewer perithecia compared with the wild-type and complemen-
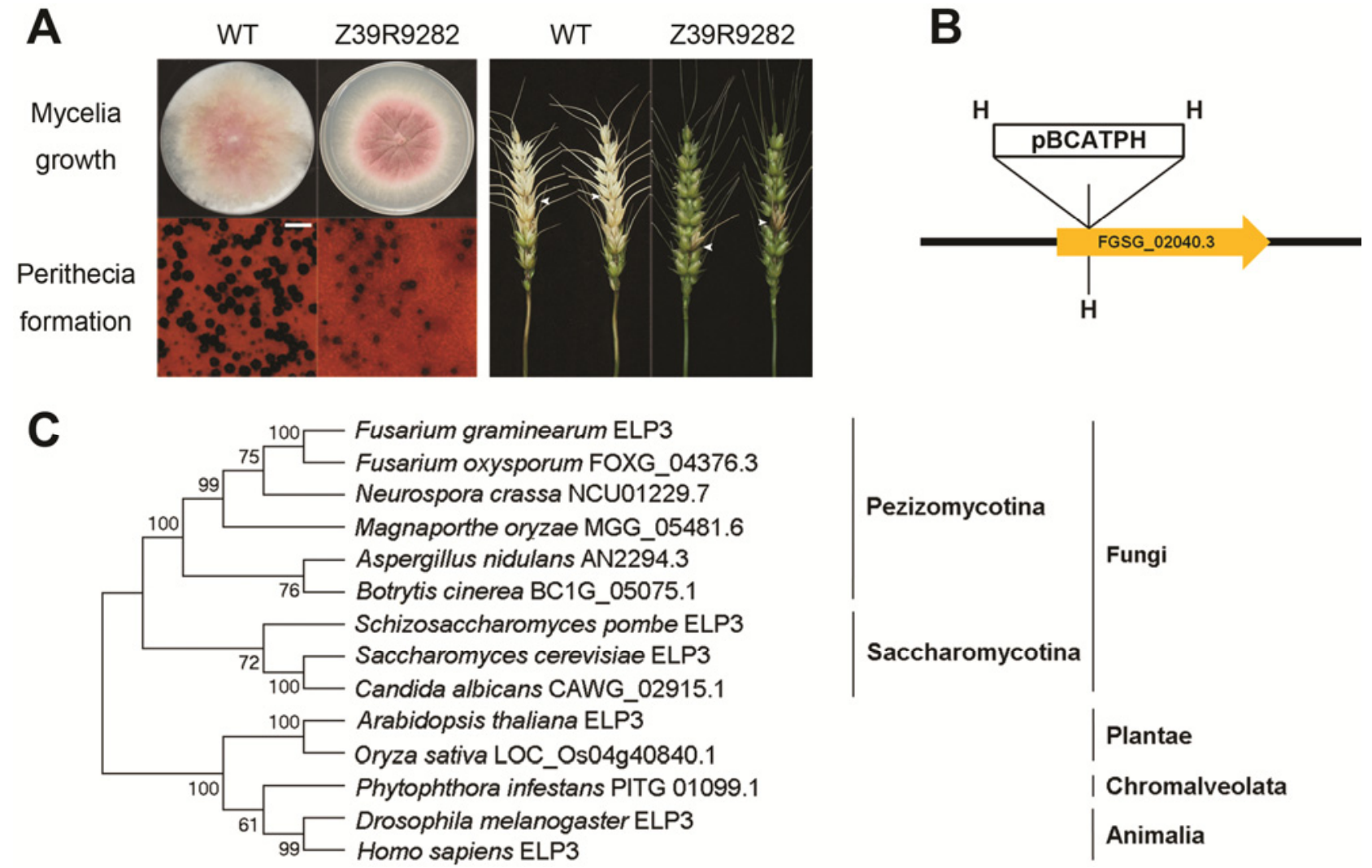

Fig. 1. Identification of elp3. A, Phenotypes of the Z39R9282 mutant. Mycelial growth on complete media (CM), perithecium formation on carrot agar, and virulence on wheat heads. Pictures were taken 5 days after inoculation on CM, 7 days after sexual induction on carrot agar, and 21 days after inoculation on wheat heads. Scale bar $=500 \mu \mathrm{m}$. Arrowheads mark the inoculated spikelets. B, Schematic showing where the pBCATPH vector inserted into the Z39R9282 genome. C, Phylogenetic tree of ELP3 orthologs in eukaryotes by ClustalW alignment. MEGA program version 5.2 was used to perform a 1,000-bootstrap phylogenetic analysis using the neighbor joining method. WT $=$ the Fusarium graminearum wild-type strain Z-3639 and $\mathrm{H}=$ HindIII . 
tation strains (Fig. 2B). Perithecia maturation was also delayed but not abolished in the elp3 deletion mutant. Seven days after sexual induction, perithecia produced asci with mature ascospores in the wild-type strain, whereas immature

A

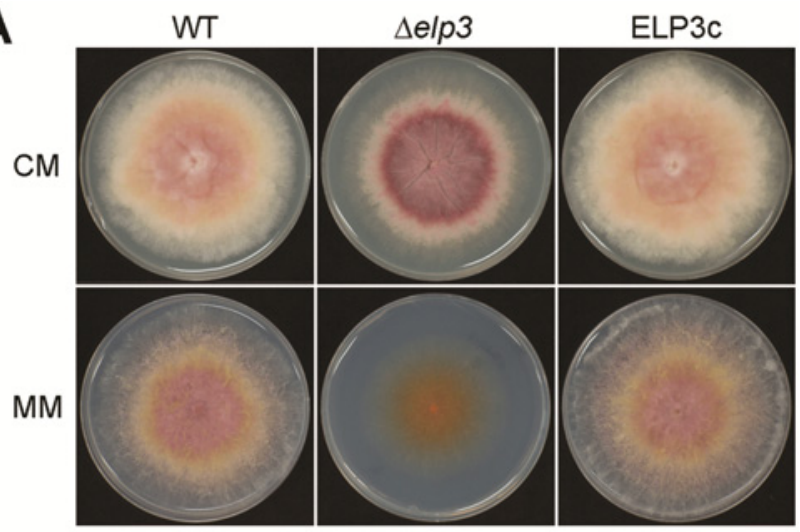

B

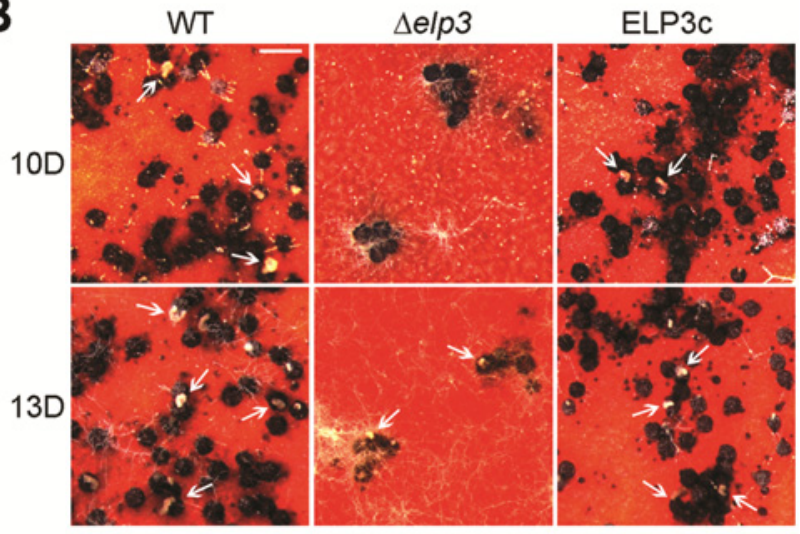

C
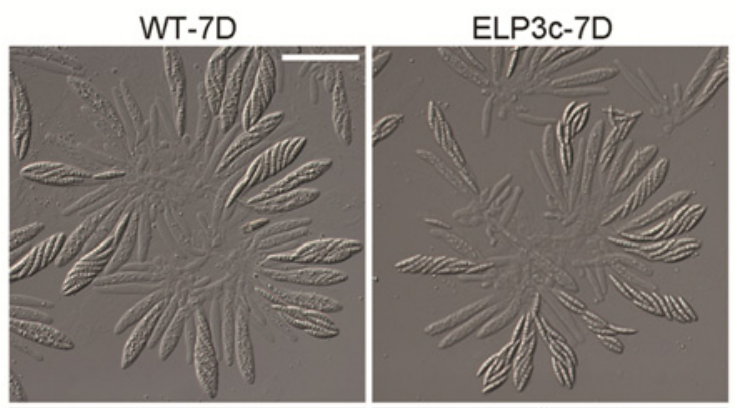

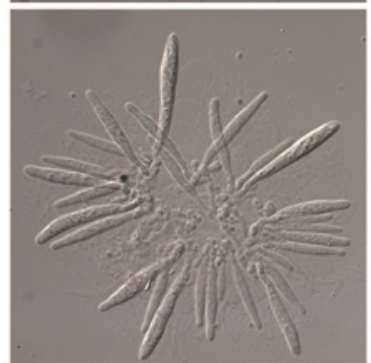

$\Delta e l p 3-7 D$

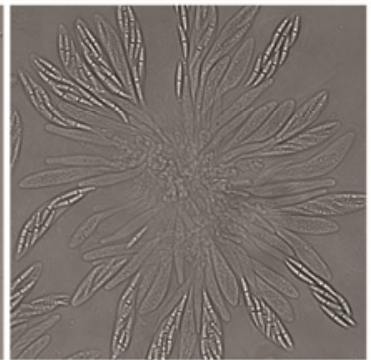

$\Delta$ elp3-10D

D
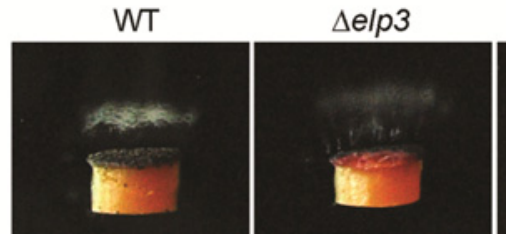

ELP3C

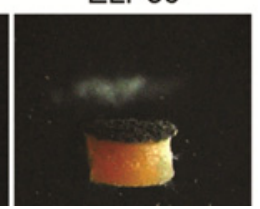

perithecia with young asci were observed in the elp3 deletion mutant. Ten days after sexual induction, several asci containing mature ascospores were observed in the elp3 deletion mutant (Fig. 2C). The elp3 deletion mutant produced ascospore cirrhi (Fig. 2B) and discharged ascospores (Fig. 2D), similar to the wild-type strain. The reduced number of discharged ascospores of the elp3 deletion mutant was due to fewer perithecia (Fig. 2D).

Although both the wild-type and the elp3 deletion mutant strains produced and discharged ascospores, their morphologies differed (Fig. 3A). To visualize the nucleus in the elp3 deletion mutant, we generated strain YJ19 (Aelp3::gen hH1:: hH1-gfp-hyg) by outcrossing mat1g (Hong et al. 2010) and the elp3 deletion mutant. In the wild-type strain, the majority of ascospores were four celled and the individual cells were uninucleate and of equal size. In contrast, the ascospores in the elp3 deletion mutant were mainly two celled and each cell tended to contain two nuclei (Fig. 3B). The mean ascospore length of the elp3 deletion mutant $(21.9 \mu \mathrm{m})$ was similar to that of the wild-type strain $(20.8 \mu \mathrm{m})$ regardless of the number of cells (Fig. 3A). The germinability of ascospores was not affected by the deletion of elp3 (Table 1). Binucleate twocelled ascospores from the elp3 deletion mutant germinated on $\mathrm{CM}$ without changes in septation (Supplementary Fig. S3).

Conidia production and germination of the elp3 deletion mutant did not differ from the wild-type and complementation strains (Table 1). The average length of conidia produced from the elp3 deletion mutant on yeast extract-malt extract agar (YMA) was approximately $20 \%$ longer than conidia produced from the wild-type strain $(P<0.01)$. The mean length of conidia from the elp3 deletion mutant was $59.2 \mu \mathrm{m}$, while that of the wild-type strain was $47.8 \mu \mathrm{m}$ (Fig. 3C). Despite the increased length of the conidia in the elp3 deletion mutant, the number of septa was similar to the wild type (Fig. 3C).

\section{Trichothecene production and virulence.}

The production of trichothecenes was analyzed in minimal media containing $5 \mathrm{mM}$ agmatine (MMA) by gas chromatography mass spectrometry. Deoxynivalenol and 15-acetyldeoxynivalenol, major trichothecenes produced in MMA, were not detected in the elp3 deletion mutant (Fig. 4A), whereas trichothecene production by the complementation strain did not differ from that of the wild-type strain. The quantitative realtime polymerase chain reaction (qRT-PCR) results showed that transcription of the trichothecene biosynthesis genes tri5 and tri6 was significantly reduced in the elp3 deletion mutant compared with the wild-type strain (Fig. 4B).

In a virulence test, 21 days after wheat head inoculation, the elp3 deletion mutant caused significantly reduced disease symptoms $(P<0.01)$, whereas the wild-type and complementation strains caused typical head blight symptoms (Fig. 4C).

Fig. 2. Vegetative growth and sexual development of Fusarium graminearum strains. A, Mycelial growth of $F$. graminearum strains. Pictures were taken 5 days after inoculation on complete media $(\mathrm{CM})$ and minimal media $(\mathrm{MM}) . \mathrm{WT}=$ the $F$. graminearum wild-type strain Z-3639, $\Delta$ elp3 = the elp3 deletion mutant (YJ17), and ELP3c = the $\Delta$ elp 3 mutant-derived strain complemented with elp3 (YJ18). B, Perithecium formation by $F$. graminearum strains. Cirrhi (indicated with white arrow) were observed in the WT and complementation strains 10 days (D) after sexual induction. Thirteen days after sexual induction, the elp 3 deletion mutants formed cirrhi. Pictures were taken 10 and 13 days after sexual induction on carrot agar. Scale bar $=500 \mu \mathrm{m}$. C, Morphologies of asci rosettes. Microscopic observations were performed 7 and 10 days (D) after sexual induction. Scale bar $=50 \mu \mathrm{m}$. D, Forcible discharge of ascospores from perithecia produced on carrot agar. A semicircular agar block covered with mature perithecia was placed on a coverslip. 
In wheat heads inoculated with the elp3 deletion mutant, the spread of the disease within a spike was blocked and only artificially inoculated spikelets showed necrosis. To visualize the spread of mycelia on wheat heads during infection, strains constitutively expressing green fluorescent protein (GFP) were injected into the center spikelets of wheat heads. Six days after inoculation with the elp3 deletion mutant that constitutively expresses GFP (YJ19), hyphae were seen throughout the rachis but failed to spread to the adjacent spikelet (Fig. 5), whereas inoculation with the wild-type GFP-expressing strain (hH1-GFP) led to the spread of hyphae to the adjacent spikelet through rachis node.

\section{Stress sensitivity assay.}

Previous studies on elp 3 in other eukaryotes revealed that it regulated the expression of stress-inducible genes (Chen et al. 2006; Han et al. 2007a; Wittschieben et al. 2000). To determine whether elp3 is required for the stress response in $F$. graminearum, strains were inoculated onto $\mathrm{CM}$ supplemented with various stress agents, including osmotic stress agents $(\mathrm{NaCl}, \mathrm{KCl}$, and D-sorbitol), an oxidative stress agent $\left(\mathrm{H}_{2} \mathrm{O}_{2}\right)$, cell-wall-damaging agents (sodium dodecyl sulfate [SDS] and Congo red), and fungicides (fludioxonil, iprodione, and benomyl), and their sensitivities to these agents were evaluated. In addition, sensitivity to high-temperature stress was tested. Hyphal growth of the elp3 deletion mutant was severely inhibited by oxidative stress compared with the wild-type strain (Fig. 6A) but none of the other stress conditions affected hyphal growth of the elp3 deletion mutant (Supplementary Figs. S4 and S5), indicating that elp3 was specifically involved in the oxidative stress response.

\section{Identification of catalase genes in $F$. graminearum.}

To investigate the expression of oxidative stress-induced genes in the elp3 deletion mutant, we first identified catalase genes in $F$. graminearum. Five putative monofunctional catalase genes (FGSG_06554.3, FGSG_06733.3, FGSG_16526.3, FGSG_02881.3, and FGSG_06596.3) were previously identified by BLASTp searches using Cryptococcus neoformans catalase sequences (Giles et al. 2006). We identified two bifunctional catalase-peroxidase genes (FGSG_02974.3 and FGSG_12369.3) by BLASTp searches in the Fusarium Comparative Database using $M$. oryzae catalase-peroxidase sequences (Skamnioti et al. 2007). We designated these seven F. graminearum catalase genes $f c a l$ to $f c a 7$ (Table 2).

Table 1. Radial growth, ascospore germination, and conidiation in Fusarium graminearum strains ${ }^{\mathrm{w}}$

\begin{tabular}{|c|c|c|c|c|}
\hline \multirow[b]{2}{*}{ Strain } & \multirow[b]{2}{*}{ Radial growth $(\mathrm{cm})^{\mathrm{x}}$} & \multirow[b]{2}{*}{ Ascospore germination $(\%)^{\mathrm{y}}$} & \multicolumn{2}{|r|}{ Conidia } \\
\hline & & & Germination $(\%)^{\mathbf{y}}$ & Production (number of conidia/ml) ${ }^{\mathrm{z}}$ \\
\hline Z-3639 & 9.8 & 69.0 & 48.3 & $2.7 \times 10^{6}$ \\
\hline YJ17 & 9.8 & 70.3 & 47.7 & $2.7 \times 10^{6}$ \\
\hline YJ18 & 10.0 & 68.3 & 50.3 & $2.6 \times 10^{6}$ \\
\hline
\end{tabular}

${ }^{\mathrm{w}}$ All data were repeated twice with three replications. Values are not significantly different $(P<0.05)$ based on a Tukey's test.

${ }^{x}$ Radial growth was measured after 5 days incubation on complete media.

${ }^{\mathrm{y}}$ Percentage was measured after $8 \mathrm{~h}$ of incubation.

${ }^{\mathrm{z}}$ Number of conidia were counted after 3 days of incubation in carboxymethyl cellulose.


D

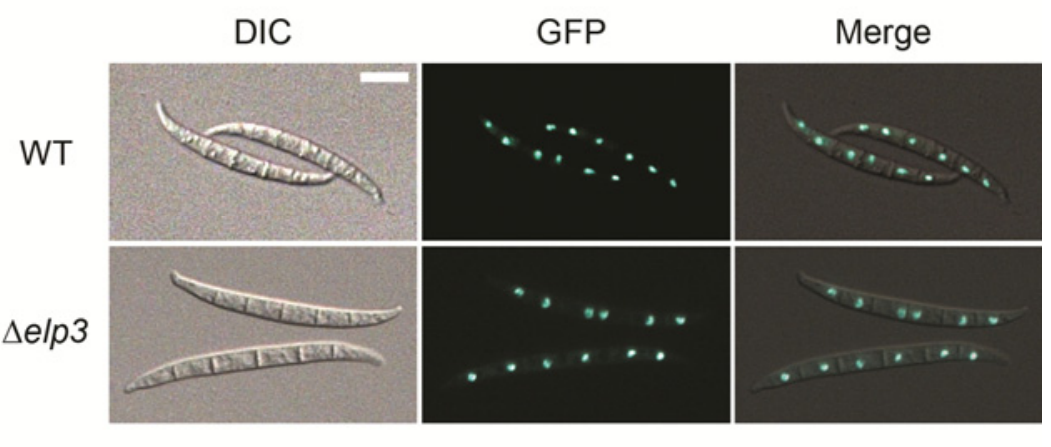

Fig. 3. Morphologies of ascospores and conidia. A, Length (dark gray) and number of septa (light gray) of ascospores from Fusarium graminearum strains. Error bars indicate the standard deviations. An asterisk indicates a significant difference $(P<0.01$, Tukey's test). WT $=$ the $F$. graminearum wild-type strain Z-3639, $\Delta$ elp3 $=$ the elp3 deletion mutant (YJ17), and ELP3c = the $\Delta$ elp3 mutant-derived strain complemented with elp3 (YJ18). B, Microscopic observations of ascospores. Fluorescent green dots represent hH1-green fluorescent protein $(\mathrm{GFP})$. DIC $=$ differential interference contrast image, GFP = fluorescence microscopy image, Merge = overlays of the DIC and fluorescence microscopy images. Scale bar $=10 \mu \mathrm{m}$. C, Length (dark gray) and number of septa (light gray) of conidia on YMA. D, Microscopic observations of conidia. Scale bar $=10 \mu \mathrm{m}$. 
Expression and functional analyses

of catalase genes in $F$. graminearum.

We performed qRT-PCR to measure transcript levels of the putative catalase genes in the wild-type and elp3 deletion mutant strains under oxidative stress. In the wild-type strain, catalase gene transcripts were upregulated in $10 \mathrm{mM} \mathrm{H}_{2} \mathrm{O}_{2}$. However, in the elp3 deletion mutant, catalase gene transcripts, except $f c a l$, were either downregulated or unchanged in $10 \mathrm{mM}$ $\mathrm{H}_{2} \mathrm{O}_{2}$ (Fig. 6B). The qRT-PCR results indicated that the elp3 deletion mutant was unable to regulate catalase gene transcription in response to oxidative stress.

To further investigate the functions of the putative catalase genes in $F$. graminearum, we generated deletion mutants of these genes through homologous recombination (Table 2; Supplementary Fig. S6) and assayed the sensitivities of these deletion mutants to $5 \mathrm{mM} \mathrm{H}_{2} \mathrm{O}_{2}$. Only the $f c a 6$ and $f c a 7$ deletion mutant strains exhibited increased sensitivities to oxidative stress compared with the wild-type strain (Fig. 6C). Hyphal growth of the $f c a 7$ deletion mutant was significantly reduced in the presence of $\mathrm{H}_{2} \mathrm{O}_{2}$, suggesting that FCA7 plays a major role in degrading extracellular $\mathrm{H}_{2} \mathrm{O}_{2}$ during vegetative growth.

We then created an $f c a 7$-overexpression strain ( $f c a 7:: g e n$ $P_{e f l \alpha}-f c a 7$, YJ28) in which $f c a 7$ expression was controlled by the elongation factor $1 \alpha$ (ef1 $\alpha$ ) promoter (Fig. 7A). qRT-PCR was used to quantify $f c a 7$ transcripts in the wild-type and $f c a 7$ overexpression strains and $f c a 7$ overexpression was confirmed in strain YJ28 (Fig. 7B). We tested the $f c a 7$-overexpression strain for sensitivity to oxidative stress and found that it was

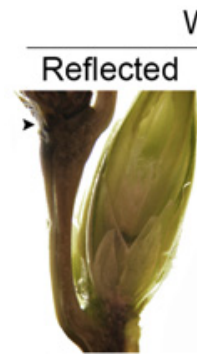

WT

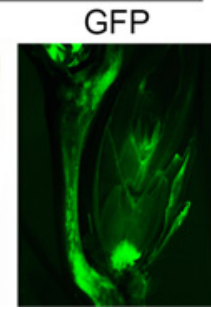

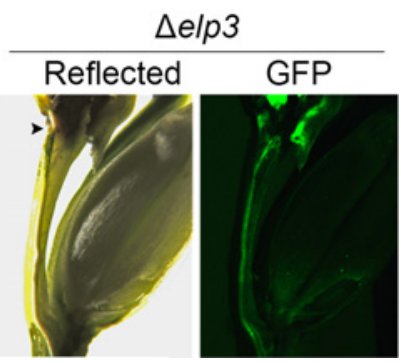

Fig. 5. Longitudinal sections of infected wheat heads. Wheat spikelets were inoculated with suspensions of conidia from green fluorescent protein (GFP)-expressing Fusarium graminearum strains. Infected wheat heads were dissected 6 days after inoculation and examined by fluorescence microscopy. Spreading of the GFP signal represents spreading of hyphae from the points of inoculation. Arrowheads mark the inoculated spikelets. WT $=$ the $F$. graminearum wild-type strain Z-3639, $\Delta$ elp $3=$ the elp3 deletion mutant YJ17, and Reflected = reflected light.

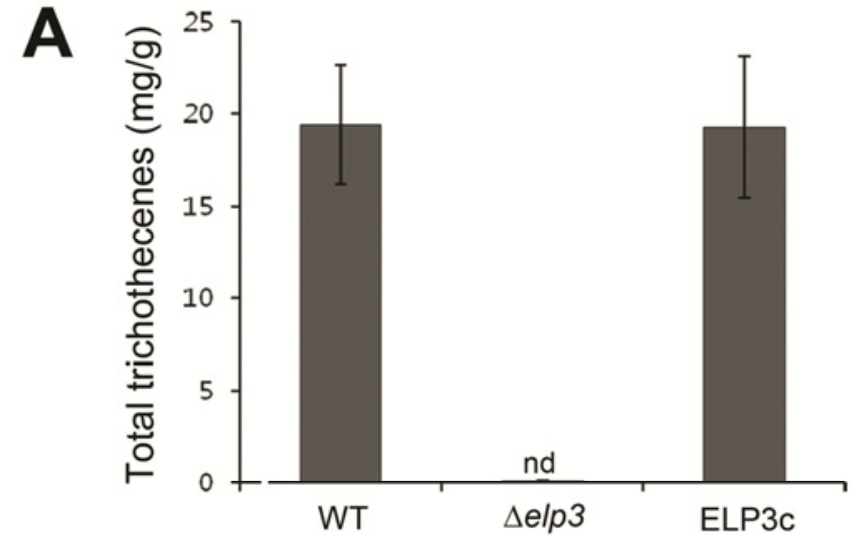

C

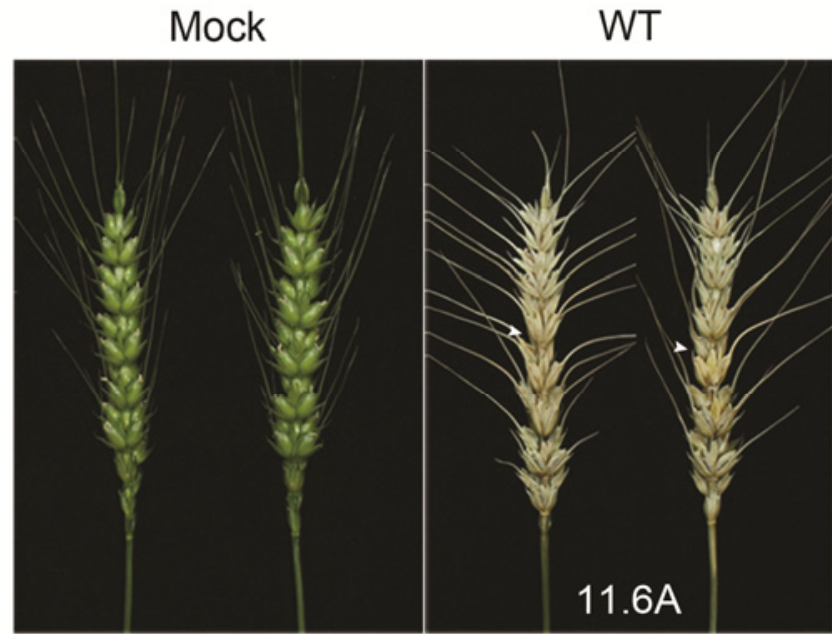

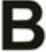

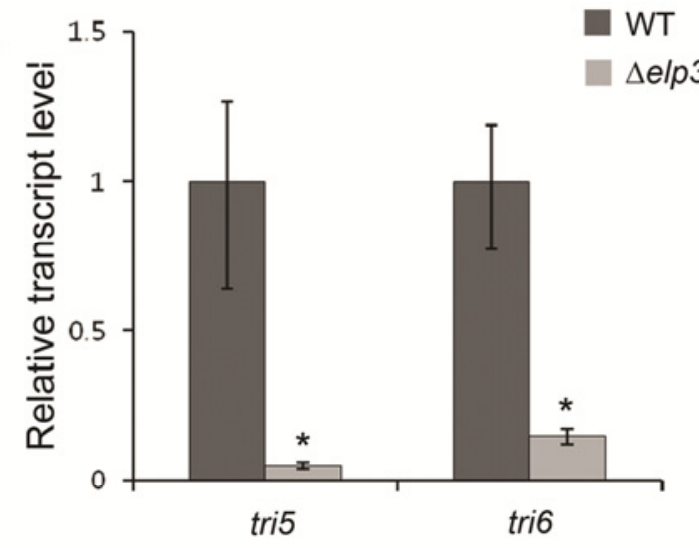

$\Delta$ elp3

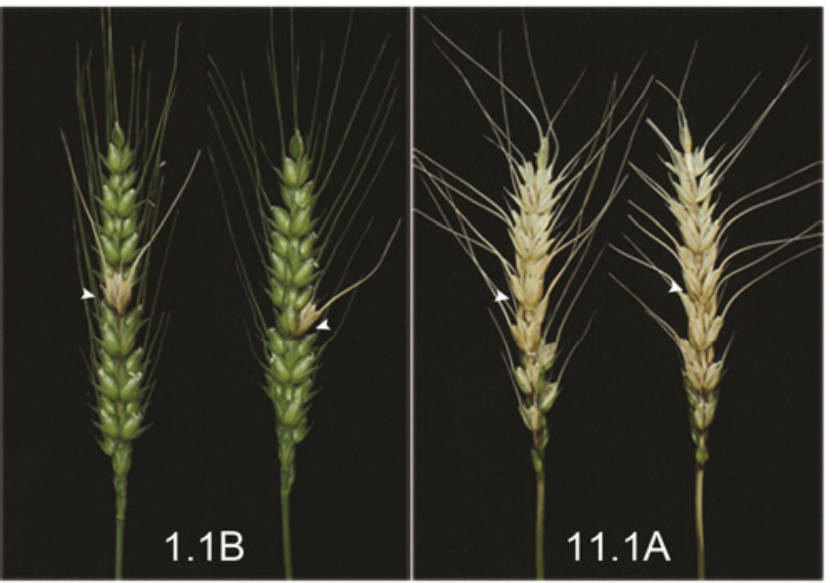

Fig. 4. Trichothecene production and virulence of Fusarium graminearum strains. A, Total trichothecene (deoxynivalenol and 15-acetyldeoxynivalenol) production by $F$. graminearum strains. Each strain was grown in minimal media containing $5 \mathrm{mM}$ agmatine (MMA) for 7 days. Trichothecenes were analyzed by gas chromatography mass spectrometry and were quantified based on the biomass of each strain. WT $=$ the $F$. graminearum wild-type strain Z-3639, $\Delta$ elp3 $=$ the elp3 deletion mutant YJ17, ELP3c $=$ the $\Delta$ elp3 mutant-derived strain complemented with elp3 $(\mathrm{YJ} 18)$, and nd $=$ not detected. B, Transcript levels of tri5 and tri6 in the WT and elp3 deletion mutant strains. Transcript levels were analyzed by quantitative real-time polymerase chain reaction 4 days after inoculation in MMA. An asterisk indicates a significant difference $(P<0.01$, Tukey's test). C, Virulence on wheat heads. The center spikelet of each wheat head was injected with $10 \mu \mathrm{l}$ of a conidial suspension, and pictures were taken 21 days after inoculation. Arrowheads indicate the inoculated spikelets. Significantly different $(P<0.01$, Tukey's test) disease indices (diseased spikelets per wheat head) are designated with different letters. Mock $=$ mock-inoculated with $0.01 \%$ of Tween 20 . 
more tolerant to $\mathrm{H}_{2} \mathrm{O}_{2}$ than the wild-type strain (Fig. 7C). To determine whether the oxidative stress sensitivity of the elp3 deletion mutant was due to the reduced expression of $f c a 7$, we generated strain YJ29 (Delp3::gen fca7::gen- $\left.P_{\text {efl } \alpha}-f c a 7\right)$ by outcrossing the heterothallic strain carrying the elp 3 mutation

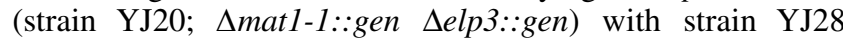
(fca7::gen-P $\left.P_{\text {efl }}-f c a 7\right)$ and confirmed the genotype of strain YJ29 by PCR. The oxidative stress sensitivity of the elp3 de- letion mutant strain was partially restored by overexpression of fca7 (Fig. 7C).

\section{Western blot analysis of histone acetylation.}

To investigate whether the deletion of elp3 affected histone $\mathrm{H} 3$ acetylation in $F$. graminearum, we measured acetylation of lysine 14 in $\mathrm{H} 3$ (H3K14Ac) in the wild-type and elp3 deletion mutant strains by Western blot analysis (Fig. 8A). We chose

Table 2. Fusarium graminearum strains used in this study

\begin{tabular}{|c|c|c|}
\hline Strain & Genotype & Reference \\
\hline Z-3639 & $F$. graminearum wild type & Bowden and Leslie 1999 \\
\hline Z39R9282 & REMI mutant & This study \\
\hline YJ17 & $\Delta e l p 3::$ gen & This study \\
\hline YJ18 & selp3::elp3-hyg & This study \\
\hline hH1-GFP & $h H 1:: h H 1-g f p-h y g$ & Hong et al. 2010 \\
\hline$\Delta m a t 1$ & $\Delta$ mat1-1::gen & Lee et al. 2003 \\
\hline mat1g & $\Delta$ mat1-1::gen hH1::hH1-gfp-hyg & Hong et al. 2010 \\
\hline YJ19 & $\Delta$ elp3::gen hH1::hH1-gfp-hyg & This study \\
\hline YJ20 & $\Delta$ mat1-1::gen $\Delta$ elp3::gen & This study \\
\hline YJ21 & $\Delta f c a l:: g e n$ & This study \\
\hline YJ22 & $\Delta f c a 2:: g e n$ & This study \\
\hline YJ23 & $\Delta f c a 3:: g e n$ & This study \\
\hline YJ24 & $\Delta f c a 4: \because g e n$ & This study \\
\hline YJ25 & $\Delta f c a 5:: g e n$ & This study \\
\hline YJ26 & $\Delta f c a 6: \because g e n$ & This study \\
\hline YJ27 & $\Delta f c a 7: \because g e n$ & This study \\
\hline YJ28 & fca $7:: g e n-P_{e f 1}-f c a 7$ & This study \\
\hline YJ29 & $\Delta$ elp3::gen fca7::gen- $P_{\text {ef } 1 \alpha^{-}}-f c a 7$ & This study \\
\hline
\end{tabular}

A

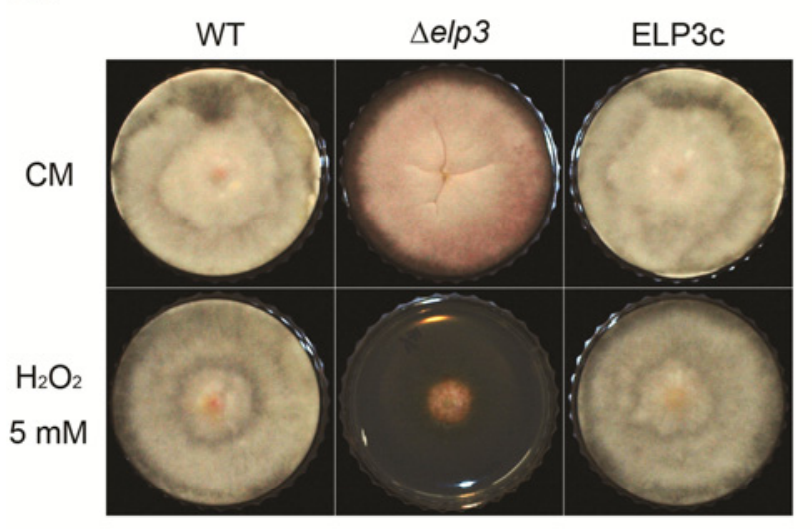

B

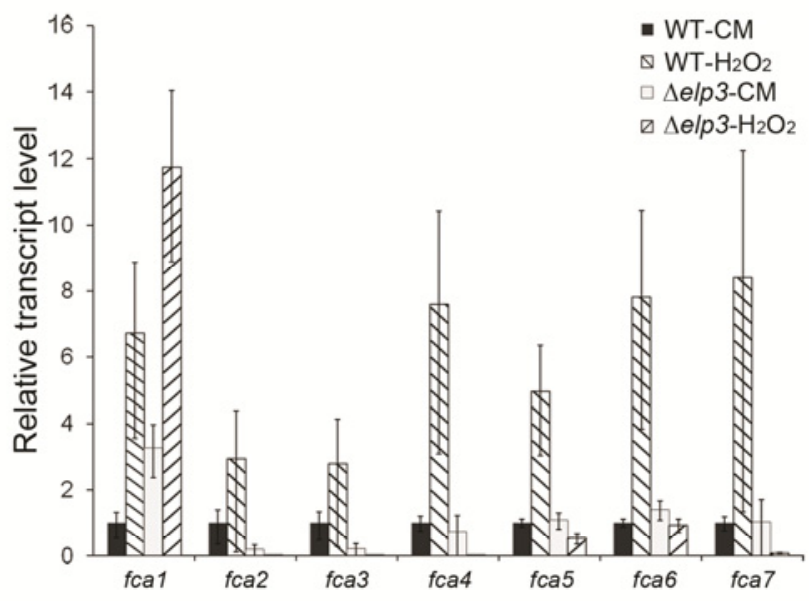

C

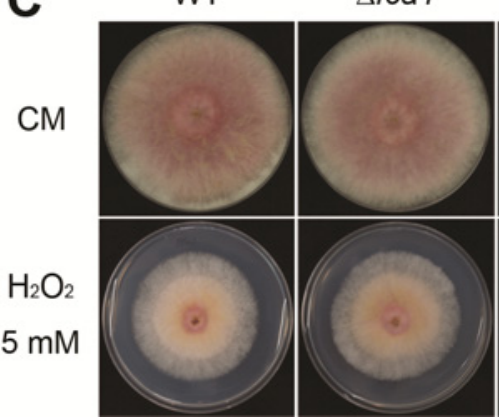

$\Delta f c a 2$

$\Delta f c a 3$

$\Delta f c a 4$

$\Delta f c a 5$

$\Delta f c a 6$

$\Delta f c a 7$
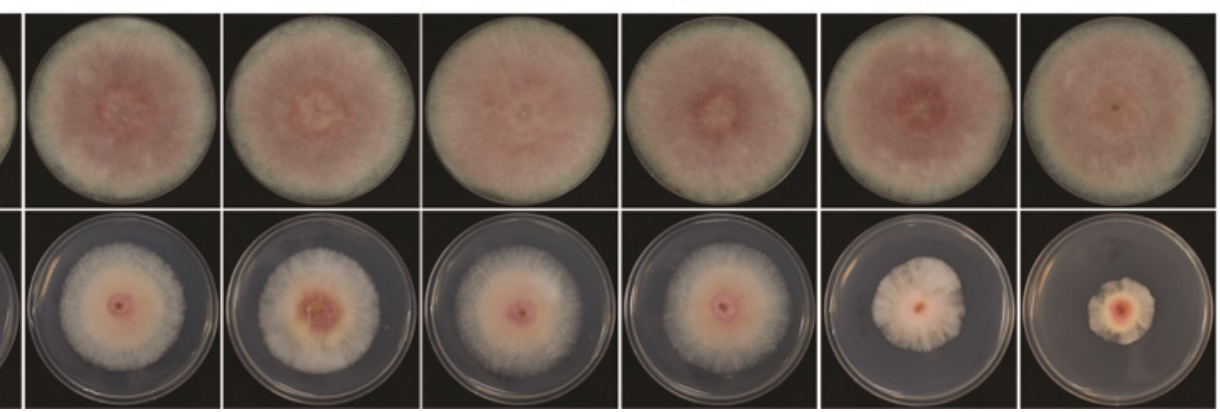

Fig. 6. Sensitivity of Fusarium graminearum strains to oxidative stress and transcriptional analyses of catalase genes. A, Mycelial growth of $F$. graminearum strains on complete media $(\mathrm{CM})$ supplemented with $5 \mathrm{mM} \mathrm{H} \mathrm{H}_{2} \mathrm{O}_{2}$. WT $=$ the $F$. graminearum wild-type strain Z-3639, $\Delta$ elp $3=$ the elp3 deletion mutant (YJ17), and ELP3c $=$ the $\Delta$ elp3 mutant-derived strain complemented with elp3 (YJ18). B, Transcript levels of catalase genes in the WT and elp3 deletion

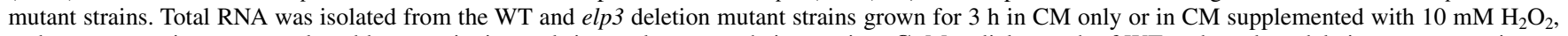
and gene transcripts were analyzed by quantitative real-time polymerase chain reaction. C, Mycelial growth of WT and catalase deletion mutant strains on CM supplemented with $5 \mathrm{mM} \mathrm{H}_{2} \mathrm{O}_{2} . \Delta f c a 1=$ the $f c a 1$ deletion mutant $(\mathrm{YJ} 21), \Delta f c a 2=$ the $f c a 2$ deletion mutant $(\mathrm{YJ} 22), \Delta f c a 3=$ the $f c a 3$ deletion mutant (YJ23), $\Delta f c a 4=$ the $f c a 4$ deletion mutant (YJ24), $\Delta f c a 5=$ the $f c a 5$ deletion mutant (YJ25), $\Delta f c a 6=$ the $f c a 6$ deletion mutant $(\mathrm{YJ} 26)$, and $\Delta f c a 7=\operatorname{the} f c a 7$ deletion mutant (YJ27). 
lysine 14 of $\mathrm{H} 3$ because, in yeast, it is one of the main acetylation sites of the Elongator complex (Winkler et al. 2002). Although relative histone acetylation level was highest 3 days after sexual induction for both the wild type and the elp3 deletion mutant, histone acetylation was markedly reduced in the elp3 deletion mutant (Fig. 8B).

\section{DISCUSSION}

In this study, we identified a gene encoding an ortholog of yeast ELP3 in $F$. graminearum using forward genetics. To elucidate the functions of ELP3 in $F$. graminearum, we created an elp3 deletion mutant and analyzed its phenotypes. We found that elp3 was involved in diverse biological processes, including sexual and asexual reproduction, trichothecene production, and virulence in $F$. graminearum. We also found that elp3 played a role in the oxidative stress response by regulating catalase gene expression.

In $F$. graminearum, hundreds of genes required for sexual and asexual reproduction must be tightly regulated in time and space (Hallen et al. 2007; Qi et al. 2006; Son et al. 2013, 2014). Histone acetylation is generally important for the regulation of gene expression and is associated with gene activation, whereas deacetylation is associated with transcriptional repres- sion (Grunstein 1997; Kuo and Allis 1998; Struhl 1998). We found that the level of histone acetylation was lower in the elp3 deletion mutant than in the wild-type strain. We previously reported that reduced histone acetylation contributed to defects in sexual development in F. graminearum (Son et al. 2011). Thus, the reduced acetylation in the elp3 deletion mutant contributed to the sexual and asexual developmental defects in the elp3 deletion mutant.

Perithecium formation is a complex cellular differentiation process mediated by diverse signaling pathways. Inappropriate expression of genes involved in cell proliferation and differentiation resulted in defects in perithecium development in $F$. graminearum (Lin et al. 2011, 2012). Deletion of elp3 affected the production and maturation of perithecia, indicating that elp3 was required for proper gene expression during perithecium formation. The elp3 deletion mutant also produced abnormal ascospores. In the wild-type strain, an ascospore develops from a single uninucleate cell. Following the first mitosis, a medial septum is formed, resulting in a two-celled ascospore. The nucleus in each cell undergoes a second mitosis, and the resulting two nuclei are immediately separated by an additional septum. Thus, fully developed ascospores contain four uninucleate cells (Headrick et al. 1988). The elp3 deletion mutant produced ascospores containing two dinucleate cells, indi-
A WT

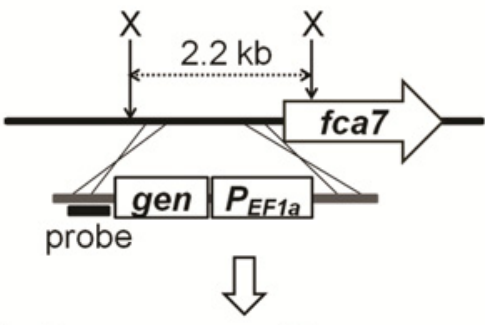

fca7OE

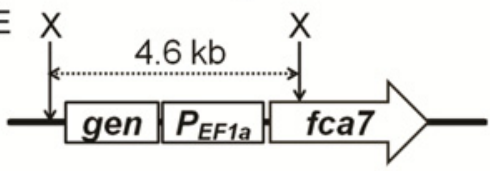

2.3

4.3



Fig. 7. Sensitivity of the $f c a 7$ overexpression strain to oxidative stress. A, The $e f l \alpha$ promoter was inserted into the natural promoter of $f c a 7$. The left panel shows how the $f c a 7$ overexpression strain was constructed and the right panel confirms insertion by Southern analysis. WT $=$ the Fusarium graminearum wild-type strain Z-3639, fca7OE = overexpression strain in which the natural $f c a 7$ promoter was replaced with the efl $\alpha$ promoter $(\mathrm{YJ} 28)$, X $=$ XalI, and gen $=$ geneticin resistance gene. Lane 1, Z-3639; lanes 2 and 3, isolates of the $f c a 7$ overexpression strain. The DNA size standards (kb) are indicated on the left of the blot. B, Transcript levels of $f c a 7$ in the WT and $f c a 7$ overexpression strains. Total RNA was isolated from the WT and $f c a 7$ overexpression strains grown for $3 \mathrm{~h}$ in complete media (CM) only or in CM supplemented with $\mathrm{H}_{2} \mathrm{O}_{2}$, and transcript levels were analyzed by quantitative real-time polymerase chain reaction. $\mathbf{C}$, Mycelial growth of fungal strains on $\mathrm{CM}$ supplemented with $5 \mathrm{mM} \mathrm{H}_{2} \mathrm{O}_{2} . \Delta$ elp $3=$ the elp3 deletion mutant $(\mathrm{YJ} 17)$ and $\Delta$ elp3-fca $7 O E=$ the elp3 deletion mutant that overexpresses $f c a 7$ (YJ29). 
cating a failure to progress through the second mitotic division. When assessing asexual development, we found that the elp3 deletion mutant produced longer conidia compared with the wild type. Defects in cell cycle progression during conidiogenesis in $F$. graminearum frequently results in elongated conidia (Min et al. 2014; Son et al. 2014). Deletion of wetA, a $F$. graminearum transcription factor known to be involved in conidiogenesis, resulted in longer conidia (Son et al. 2014). Taken together, these results indicate that the defects in sexual and asexual development of the elp 3 deletion mutant result from impairments in cell cycle progression.

The elp3 deletion mutant exhibited reduced virulence on wheat heads, and several $F$. graminearum genetic studies have shown that trichothecenes are virulence factors for plant disease (Desjardins et al. 1996; Maier et al. 2006; Proctor et al. 1995). Thus, we hypothesized that reduced trichothecene production might contribute to the reduced virulence of the elp3 deletion mutant. In the elp3 deletion mutant, the transcript levels of the trichothecene biosynthesis genes tri5 and tri6 were significantly lower than in the wild-type strain. These observations suggested that elp3 played a role in virulence by regulating trichothecene biosynthesis gene expression.

In many organisms, ELP3 has been found to regulate genes involved in stress responses (DeFraia et al. 2013; Han et al. 2007a; Nelissen et al. 2005; Walker et al. 2011; Wittschieben et al. 2000). In yeast, an elp3 deletion mutant showed a delayed response to high temperature and salt stress as well as reduced activation of several genes, including gall-10 and pho5, which are required for survival under stressful growth conditions (Wittschieben et al. 2000). In plants, ELP3 has been found to be required for basal plant immunity (DeFraia et al. 2013) and, in human cells, ELP3 regulates hsp70 expression by acetylating histones $\mathrm{H} 3$ and $\mathrm{H} 4$ in heat-shock conditions (Han et al. 2007a). Although the role of elp 3 in the stress response is conserved in eukaryotes, the subset of stressinducible genes has diverged during evolution (Creppe and Buschbeck 2011).

We tested the sensitivity of the elp3 deletion mutant to diverse stress conditions and found that $F$. graminearum elp3 was mainly involved in the oxidative stress response. Oxidative stress is a stress commonly encountered by phytopathogenic fungi. During infection, fungal pathogens encounter toxic environments generated by the hosts' defense mechanisms. The oxidative burst, a rapid production of reactive oxygen species (ROS), is one of the host's earliest responses to pathogen infection (Torres et al. 2006; Wojtaszek 1997). Several pathogens produce ROS-scavenging enzymes to protect themselves from the plant-derived oxidative stress (Schouten et al. 2002); catalase, which metabolizes $\mathrm{H}_{2} \mathrm{O}_{2}$, is one of these antioxidant enzymes. We found that, in F. graminearum, elp3 was required for catalase gene expression under oxidative stress conditions.

Seven putative catalase genes, $f c a l$ to $f c a 7$, have been identified in F. graminearum through BLAST searches, and we generated deletion mutants for all seven genes. Among these mutants, only the $f c a 6$ and $f c a 7$ deletion mutants showed increased sensitivity to extracellular $\mathrm{H}_{2} \mathrm{O}_{2}$. Deletion of the other catalase genes did not affect sensitivity to oxidative stress, which may be due to the overlapping and redundant antioxidant functions of these genes. In $C$. neoformans, single and quadruple catalase mutants did not exhibit oxidative-stress phenotypes, indicating that $C$. neoformans possessed a robust and redundant antioxidant defense system (Giles et al. 2006). Because $f c a 7$ primarily contributed to the extracellular $\mathrm{H}_{2} \mathrm{O}_{2}$ resistance phenotype, we constructed an elp 3 deletion mutant that overexpressed $f c a 7$. We found that the oxidative stress sensitivity of the elp3 deletion mutant was partially comple- mented by overexpression of $f c a 7$. Thus, elp3 may confer resistance to oxidative stress by regulating the expression of fca7.

In this study, we observed that the elp 3 deletion mutant had reduced catalase activity under oxidative stress and exhibited reduced virulence on wheat heads. In Claviceps purpurea, deletion of the transcription factor cptfl, a general regulator of catalase activity, resulted in reduced virulence (Nathues et al. 2004). In M. oryzae, it was reported that the catalase gene $c a t B$ was involved in its pathogenicity (Skamnioti et al. 2007). In addition, the catalase-peroxidase gene $c p x B$ in $M$. oryzae played a role in fungal defense against $\mathrm{H}_{2} \mathrm{O}_{2}$ accumulated in epidermal cells during early infection (Tanabe et al. 2011).

In a previous study, $F$. graminearum HDAC genes hdfl, $h d f 2$, and $h d f 3$ were characterized ( $\mathrm{Li}$ et al. 2011). The deletion of the HDAC genes led to the opposite results for pigmentation, conidial morphology, and response to oxidative stress than the elp3 deletion. Unlike the $h d f 1$ deletion mutant, which formed whitish colonies on potato dextrose agar ( $\mathrm{Li}$ et al. 2011), the elp3 deletion mutant displayed more red pigmentation than the wild-type strain. The conidia produced by the elp3 deletion mutant were longer than those produced by the wild-type strain, whereas the $\Delta h d f 1 \Delta h d f 2$ double mutant produced shorter conidia (Li et al. 2011). In the hdfl deletion mutant, sensitivity to $\mathrm{H}_{2} \mathrm{O}_{2}$ was reduced and the putative catalase genes were upregulated ( $\mathrm{Li}$ et al. 2011), whereas the elp3 deletion mutant was more sensitive to $\mathrm{H}_{2} \mathrm{O}_{2}$ and catalase gene expression was reduced. Because HAT and HDAC have opposite functions (Kuo and Allis 1998), these observations raised the possibility that elp3 cooperated with HDAC genes to maintain the appropriate level of histone acetylation during these biological processes. In yeast, the phenotype of a $\Delta e l p 3 \Delta$ gcn 5 double mutant was partially restored by mutation of specific

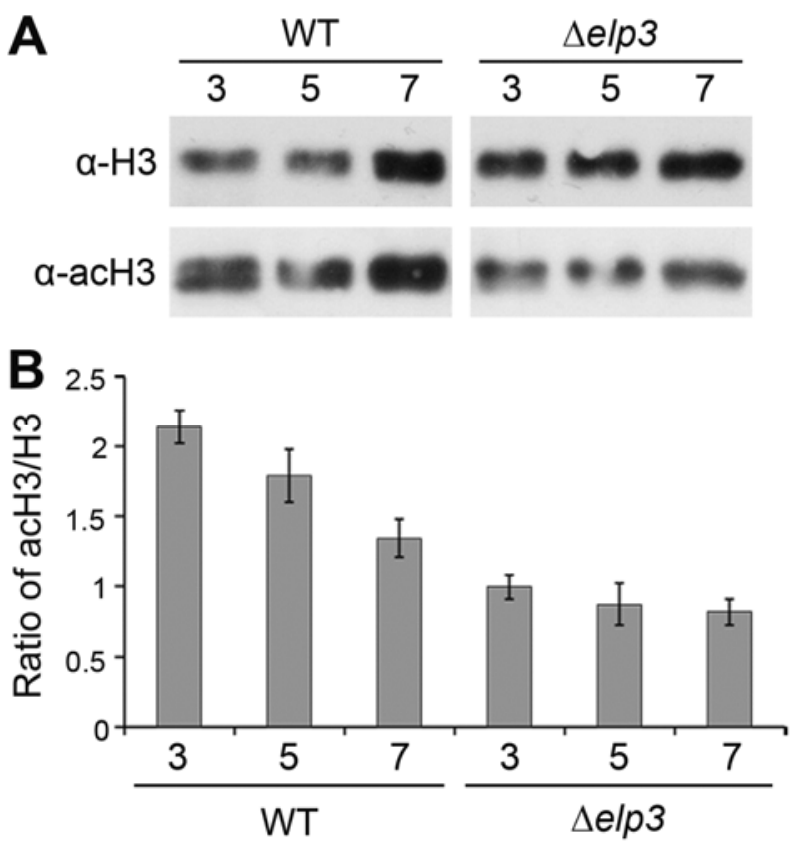

Fig. 8. Western blot of whole cell extracts of Fusarium graminearum strains. A, Whole cell extracts were prepared from carrot agar cultures. $\mathrm{WT}=$ the $F$. graminearum wild-type strain Z-3639, $\Delta$ elp $3=$ the elp3 deletion mutant (YJ17), $\alpha-\mathrm{H} 3$ = primary antibody against histone $\mathrm{H} 3$, and $\alpha$ $\mathrm{acH} 3$ = primary antibody against histone $\mathrm{H} 3$ acetylated at Lys14. Numbers above the blots indicate the number of days of incubation after sexual induction. B, Levels of $\alpha-\mathrm{acH} 3$ and $\alpha-\mathrm{H} 3$ binding were quantified using Image Studio software, and the data are presented as the ratio of $\alpha$ acH3/ $\alpha-\mathrm{H} 3$ binding. Error bars represent the standard deviations of three independent experiments. 
HDAC genes, demonstrating the balance between histone acetylation and deacetylation (Wittschieben et al. 2000).

In conclusion, we have shown that $F$. graminearum elp 3 is involved in diverse biological processes, including sexual and asexual reproduction, secondary metabolite production, virulence, and the oxidative stress response. This article demonstrated that the defects in the elp3 deletion mutant were associated with reduced histone acetylation and demonstrated that elp3 was required for activation of genes involved in oxidative stress, a stress that pathogens encounter upon plant invasion.

\section{MATERIALS AND METHODS}

\section{Fungal strains and culture media.}

The F. graminearum wild-type strain Z-3639 (Bowden and Leslie 1999) and mutants derived from this strain were used in this study (Table 2). All strains were stored as conidial suspensions in $20 \%$ glycerol at $-70^{\circ} \mathrm{C}$. Culture media used in this study were prepared following the Fusarium laboratory manual (Leslie and Summerell 2006). For conidia production, carboxymethyl cellulose (CMC) liquid culture media (Cappellini and Peterson 1965) and YMA media (Harris 2005) were used. MMA was used for trichothecene production (Gardiner et al. 2009).

\section{Nucleic acid manipulations, primers, PCR conditions, and DNA sequencing.}

Genomic DNA was extracted from mycelia powder as previously described (Leslie and Summerell 2006). Total RNA was extracted from mycelia in liquid nitrogen using the Easy-Spin Total RNA Extraction Kit (Intron Biotech, Seongnam, Korea). Standard techniques were used for restriction endonuclease digestion, Southern hybridization with ${ }^{32} \mathrm{P}$-labeled probes, agarose gel electrophoresis, and plasmid rescue (Sambrook and Russell 2001). The PCR primers used in this study (Supplementary Table S1) were synthesized by an oligonucleotide synthesis facility (Bionics, Seoul, Korea). PCR was performed according to manufacturer's instructions (Takara Bio Inc., Ostu, Japan). DNA sequencing was performed at the National Instrumentation Center for Environmental Management (Seoul National University, Seoul, Korea) and sequences were compared against the Fusarium Comparative Database at the Broad Institute.

\section{Genetic manipulations and fungal transformations.}

The double-joint (DJ) PCR method was used to generate fusion PCR products for the creation of targeted gene deletion, complementation, and overexpression strains (Yu et al. 2004). To create deletion strains, gen was amplified from pII99 (Namiki et al. 2001), and the $5^{\prime}$ and $3^{\prime}$ flanking regions of target genes were amplified from the wild-type strain. The three amplicons ( $5^{\prime}$ flanking, 3' flanking, and gen) were fused by DJ PCR as previously described (Hong et al. 2010). For complementation of the elp3 deletion mutant, a wild-type copy of elp3 was fused with hyg from pBCATPH (Gritz and Davies 1983). The fusion construct was transformed into the deletion mutant as previously described (Han et al. 2007b). To construct the $f c a 7$ overexpression mutant, a fusion construct containing the $5^{\prime}$ flanking region of $f c a 7$, the $f c a 7 \mathrm{ORF}$, and the gen- $P_{\text {efl } \alpha}$ region from pSKGEN (Lee et al. 2011) was created and transformed into the wild-type strain.

\section{Conidia production and germination.}

Fungal strains were incubated in $50 \mathrm{ml}$ of $\mathrm{CM}$ for $72 \mathrm{~h}$ at $25^{\circ} \mathrm{C}$ on a rotary shaker $(150 \mathrm{rpm})$. Mycelia were harvested and washed twice with sterile distilled water. To induce conidiation, harvested mycelia were spread onto YMA and incubated for $48 \mathrm{~h}$ at $25^{\circ} \mathrm{C}$ under near-UV light (wavelength: $365 \mathrm{~nm}$;
HKiv Import \& Export Co., Ltd., Xiamen, China). Conidia were collected in sterile distilled water, filtered through cheesecloth, washed with sterile distilled water, and collected by centrifugation $\left(5,000 \mathrm{rpm}, 25^{\circ} \mathrm{C}, 5 \mathrm{~min}\right)$. Conidia production was measured by counting the number of conidia produced after inoculating $5 \mathrm{ml}$ of CMC with $10 \mu \mathrm{l}$ of conidia suspension $\left(10^{5}\right.$ conidia/ml $)$ and incubating for $72 \mathrm{~h}$ at $25^{\circ} \mathrm{C}$ on a rotary shaker $(150 \mathrm{rpm})$. The rate of conidia germination was determined as previously described (Lee et al. 2009b). In brief, $10 \mathrm{ml}$ of CM was inoculated with $1 \mathrm{ml}$ of conidia suspension $\left(10^{6}\right.$ conidia/ml $)$ and incubated for $2,4,6,8$, and $12 \mathrm{~h}$. The number of germinating conidia was counted at each time point.

\section{Sexual crosses.}

For self-fertilizations, fungal strains were grown on carrot agar plates at $25^{\circ} \mathrm{C}$ for 5 days. The mycelia grown on carrot agar plates were mock fertilized with $700 \mu \mathrm{l}$ of a $2.5 \%$ Tween 60 solution (Leslie and Summerell 2006) to induce sexual reproduction. After sexual induction, cultures were incubated under near-UV light (wavelength: $365 \mathrm{~nm}$; HKiv Import \& Export Co., Ltd.) at $25^{\circ} \mathrm{C}$. Perithecia and ascospores were observed 7 to 10 days after fertilization. For outcrosses, mycelia of heterothallic female strains grown on carrot agar plates for 5 days were fertilized with $1 \mathrm{ml}$ of a male strain conidia suspension. After sexual induction, cultures were incubated under near-UV light at $25^{\circ} \mathrm{C}$ for 7 days. Ascospores were discharged onto $2 \%$ water agar plates. Ascospores were isolated and purecultured in CM.

\section{Ascospore discharge and germination.}

Ascospore discharge was observed using small acrylic chambers ( 1 by 2.5 by $5 \mathrm{~cm}$ ) as previously described (Trail et al. 2005). A semicircular agar block ( $9.5 \mathrm{~mm}$ in diameter) covered with mature perithecia was placed on a coverslip and incubated in the chamber for $24 \mathrm{~h}$.

Ascospore germination tests were performed as previously described (Lee et al. 2009b). Ascospores were harvested by placing the carrot agar upside down and allowing the mature perithecia to discharge ascospores on petri dish covers. Ascospore suspension $\left(1 \mathrm{ml}\right.$ at $10^{6}$ conidia/ml) was incubated in 10 $\mathrm{ml}$ of $\mathrm{CM}$ for $2,4,6,8$, and $12 \mathrm{~h}$. The number of germinating ascospores was counted at each time point. The experiments were performed twice with three replicates.

\section{Virulence assays and trichothecene analysis.}

Virulence tests were performed using the susceptible wheat 'Eunpamil' as previously described (Lee et al. 2009a). Conidial suspensions $\left(10 \mu \mathrm{l}\right.$ at $10^{6}$ conidia/ml) harvested from CMC were injected into center spikelets of wheat heads at midanthesis. After inoculation, plants were incubated in a humidity chamber for 3 days and then transferred to a greenhouse. Spikelets displaying symptoms of FHB were counted 21 days after inoculation.

Six days after inoculation, wheat spikes infected with GFPtagged strains were evaluated. Freehand longitudinal sections of spikes were cut using a clean scalpel (Baldwin et al. 2010). Sectioned spikes were viewed with reflected light and fluorescent light (excitation 470 and emission 525) using a SteREO Lumar V12 microscope (Carl Zeiss, Oberkochen, Germany).

Trichothecene production was measured as previously described (Son et al. 2011). In brief, MMA cultures were extracted with ethyl acetate and the extracts were dehydrated. Each dehydrated extract was derivatized with trimethylsilylating reagent (BSA + TMCS + TMSI, 3:2:3; Supelco, Bellefonte, PA, U.S.A.) and analyzed on a Shimadzu QP-5000 GC-MA (Shimadzu, Kyoto, Japan) using the selective ion-monitoring mode as previously described (Seo et al. 1996). 


\section{Stress sensitivity assays.}

To evaluate the effects of diverse stressors on mycelial growth, agar plugs from actively growing cultures were transferred to $\mathrm{CM}$ agar plates supplemented with osmotic stress agents (1.5 M D-sorbitol, $1.2 \mathrm{M} \mathrm{NaCl}$, and $1.2 \mathrm{M} \mathrm{KCl}$ ), an oxidative stress agent $\left(5 \mathrm{mM} \mathrm{H}_{2} \mathrm{O}_{2}\right)$, cell-wall-damaging agents (SDS at $5 \mathrm{mg} /$ liter and Congo red at $60 \mathrm{mg} / \mathrm{liter}$ ), and fungicides (fludioxonil at $0.023 \mathrm{mg} / \mathrm{liter}$, iprodione at 8.6 $\mathrm{mg} / \mathrm{liter}$, and benomyl at $0.65 \mathrm{mg} / \mathrm{liter}$ ). Plates were incubated at $25^{\circ} \mathrm{C}$ for 5 days. For the temperature sensitivity test, the wild-type, elp3 deletion mutant, and complementation strains were grown in $\mathrm{CM}$ at 25,30 , and $32^{\circ} \mathrm{C}$ for 5 days in the dark. At least two independent tests were performed for each assay and each strain was tested in triplicate.

\section{Identification of catalase genes $\boldsymbol{F}$. graminearum.}

To identify putative catalase and catalase-peroxidase genes in $F$. graminearum, a BLASTp search with $M$. oryzae catalase and catalase-peroxidase sequences (Skamnioti et al. 2007) was performed in the Fusarium Comparative Database.

\section{qRT-PCR analysis.}

Total RNA was extracted from wild-type and elp3 deletion mutant strains grown for $3 \mathrm{~h}$ in $\mathrm{CM}$ only or in CM supplemented with $10 \mathrm{mM} \mathrm{H}_{2} \mathrm{O}_{2}$. cDNA was synthesized from the total RNA using SuperScriptIII reverse transcriptase (Invitrogen, Carlsbad, CA, U.S.A.). qRT-PCR was performed using SYBR Green Super mix (Bio-Rad, Hercules, CA, U.S.A.), a 7500 real-time PCR system (Applied Biosystems, Foster City, CA, U.S.A.), and primer pairs specific for catalase genes. The cyclophilin gene ( $C y P 1$; FGSG_07439.3) was used as the endogenous control for normalization (Kwon et al. 2009). qRT-PCRs were performed three times, and the transcript level of each target gene was calculated as previously described (Livak and Schmittgen 2001).

To analyze transcription of the trichothecene biosynthesis genes tri5 and tri6, we incubated conidia from wild-type and elp3 deletion mutant strains in MMA media for 5 days, isolated total RNA from each strain, and performed qRT-PCR as described above.

\section{Microscopic observations.}

Spore morphology was observed by differential interference contrast (DIC) microscopy. DIC images were obtained with a DE/Axio Imager A1 microscope (Carl Zeiss) equipped with an UPlanApo $\times 100$ objective lens (1.3 numerical aperture; Carl Zeiss). AxioVision software (release 4.7; Carl Zeiss) was used to measure spore dimensions. Chitin staining was conducted by adding Calcofluor white stock solution $(10 \mathrm{mg} / \mathrm{ml}$; Sigma, St. Louis) to ascospore samples on slide glasses as previously described (Son et al. 2012). Ascospores were observed using filter set 49 (excitation 356; emission 445/50). To visualize GFP expression in spores, microscopy was performed with the DE/Axio Imager A1 microscope (Carl Zeiss) using the $38 \mathrm{H}$ (excitation 470/40; emission 525/50) filter set.

\section{Western blotting.}

Whole cell extracts (WCE) were prepared as previously described (Son et al. 2011). In brief, mycelia harvested from carrot agar cultures were ground in liquid nitrogen and extracted with $1 \mathrm{ml}$ of breaking buffer $(50 \mathrm{mM}$ Tris at $\mathrm{pH} 8.4,192 \mathrm{mM}$ glycine, and $0.1 \%$ SDS) supplemented with $1 \mathrm{mM}$ phenylmethylsulfonyl fluoride, a protease inhibitor. WCE were separated on $15 \%$ SDS polyacrylamide gels and transferred to nitrocellulose membranes (Amersham Pharmacia Biotech, Piscataway, NJ, U.S.A.). Western blot analyses were performed using chemiluminescence (Amersham ECL Select Western Blotting Detec- tion Reagent; Amersham Pharmacia Biotech) according to the manufacturer's instructions. Primary rabbit anti-histone H3 (Cell Signaling Technology, Beverly, MA, U.S.A.) and antiacetyl-Lys-4 H3 antibodies (07-353; Upstate Biotechnology, Inc., Lake Placid, NY, U.S.A.) were used at a 1:1,000 dilution. Anti-rabbit secondary antibody conjugated to horseradish peroxidase (Amersham Pharmacia Biotech) was used at a 1:4,000 dilution. The blocking buffer was composed of $5 \%$ dry nonfat milk in $0.1 \%$ Tween 20 -Tris-buffered saline ( $\mathrm{pH} 7.6$ ). The detection method used was chemiluminescence and bands were visualized and quantified with Image Studio software (version 2.0; Li-Cor Biosciences, Lincoln, NE, U.S.A.).

\section{ACKNOWLEDGMENTS}

This work was supported by the National Research Foundation of Korea grants funded by the Korea government (Ministry of Science, ICT and Future Planning numbers 2008-0061897 and 2013R1A6A3A04059121).

\section{LITERATURE CITED}

Baldwin, T. K., Urban, M., Brown, N., and Hammond-Kosack, K. E. 2010 A role for topoisomerase I in Fusarium graminearum and F. culmorum pathogenesis and sporulation. Mol. Plant-Microbe Interact. 23:566-577.

Bölker, M., Böhnert, H. U., Braun, K. H., Görl, J., and Kahmann, R. 1995. Tagging pathogenicity genes in Ustilago maydis by restriction enzymemediated integration (REMI). Mol. Gen. Genet. 248:547-552.

Bowden, R. L., and Leslie, J. F. 1999. Sexual recombination in Gibberella zeae. Phytopathology 89:182-188.

Cappellini, R. A., and Peterson, J. L. 1965. Macroconidium formation in submerged cultures by a non-sporulating strain of Gibberella zeae. Mycologia 57:962-966.

Carrozza, M. J., Utley, R. T., Workman, J. L., and Côté, J. 2003. The diverse functions of histone acetyltransferase complexes. Trends Genet. 19:321-329.

Chen, Z., Zhang, H., Jablonowski, D., Zhou, X., Ren, X., Hong, X., Schaffrath, R., Zhu, J.-K., and Gong, Z. 2006. Mutations in ABO1/ELO2, a subunit of holo-Elongator, increase abscisic acid sensitivity and drought tolerance in Arabidopsis thaliana. Mol. Cell. Biol. 26:6902-6912.

Creppe, C., and Buschbeck, M. 2011. Elongator: An ancestral complex driving transcription and migration through protein acetylation. J. Biomed. Biotechnol. 2011:924898.

DeFraia, C. T., Wang, Y., Yao, J., and Mou, Z. 2013. Elongator subunit 3 positively regulates plant immunity through its histone acetyltransferase and radical S-adenosylmethionine domains. BMC Plant Biol. 13:102.

Desjardins, A. E., and Proctor, R. H. 2007. Molecular biology of Fusarium mycotoxins. Int. J. Food Microbiol. 119:47-50.

Desjardins, A. E., Proctor, R. H., Bai, G., McCormick, S. P., Shaner, G., Buechley, G., and Hohn, T. M. 1996. Reduced virulence of trichothecene-nonproducing mutants of Gibberella zeae in wheat field tests. Mol. Plant-Microbe Interact. 9:775-781.

Gardiner, D. M., Kazan, K., and Manners, J. M. 2009. Novel genes of Fusarium graminearum that negatively regulate deoxynivalenol production and virulence. Mol. Plant-Microbe Interact. 22:1588-1600.

Giles, S. S., Stajich, J. E., Nichols, C., Gerrald, Q. D., Alspaugh, J. A., Dietrich, F., and Perfect, J. R. 2006. The Cryptococcus neoformans catalase gene family and its role in antioxidant defense. Eukaryot. Cell 5:1447-1459.

Gritz, L., and Davies, J. 1983. Plasmid-encoded hygromycin B resistance: The sequence of hygromycin B phosphotransferase gene and its expression in Escherichia coli and Saccharomyces cerevisiae. Gene 25:179188.

Grunstein, M. 1997. Histone acetylation in chromatin structure and transcription. Nature 389:349-352.

Hallen, H. E., Huebner, M., Shiu, S.-H., Güldener, U., and Trail, F. 2007. Gene expression shifts during perithecium development in Gibberella zeae (anamorph Fusarium graminearum), with particular emphasis on ion transport proteins. Fungal Genet. Biol. 44:1146-1156.

Han, Q., Hou, X., Su, D., Pan, L., Duan, J., Cui, L., Huang, B., and Lu, J. 2007a. HELP3 subunit of the Elongator complex regulates the transcription of HSP70 gene in human cells. Acta Biochim. Biophys. Sin. 39:453-461.

Han, Y.-K., Lee, T., Han, K.-H., Yun, S.-H., and Lee, Y.-W. 2004. Functional analysis of the homoserine O-acetyltransferase gene and its identification as a selectable marker in Gibberella zeae. Curr. Genet. 46:205-212. 
Han, Y.-K., Kim, M.-D., Lee, S.-H., Yun, S.-H., and Lee, Y.-W. 2007b. A novel F-box protein involved in sexual development and pathogenesis in Gibberella zeae. Mol. Microbiol. 63:768-779.

Harris, S. D. 2005. Morphogenesis in germinating Fusarium graminearum macroconidia. Mycologia 97:880-887.

Hawkes, N. A., Otero, G., Winkler, G. S., Marshall, N., Dahmus, M. E., Krappmann, D., Scheidereit, C., Thomas, C. L., Schiavo, G., and Erdjument-Bromage, H. 2002. Purification and characterization of the human Elongator complex. J. Biol. Chem. 277:3047-3052.

Headrick, J. M., Glawe, A., and Pataky, J. K. 1988. Ascospore polymorphism in Gibberella zeae. Mycologia 80:679-684.

Hong, S.-Y., So, J., Lee, J., Min, K., Son, H., Park, C., Yun, S.-H., and Lee, Y.-W. 2010. Functional analyses of two syntaxin-like SNARE genes, GzSYN1 and GzSYN2, in the ascomycete Gibberella zeae. Fungal Genet. Biol. 47:364-372.

Kim, S., Park, S.-Y., Kim, K.S., Rho, H.-S., Chi, M.-H., Choi, J., Park, J., Kong, S., Park, J., Goh, J., and Lee, Y.-H. 2009. Homeobox transcription factors are required for conidiation and appressorium development in the rice blast fungus Magnaporthe oryzae. PLoS Genet. 5:e1000757.

Kuo, M.-H., and Allis, C. D. 1998. Roles of histone acetyltransferases and deacetylases in gene regulation. BioEssays 20:615-626.

Kwon, S.-J., Cho, S.-Y., Lee, K.-M., Yu, J., Son, M., and Kim, K.-H. 2009. Proteomic analysis of fungal host factors differentially expressed by Fusarium graminearum infected with Fusarium graminearum virusDK21. Virus Res. 144:96-106.

Lee, J., Lee, T., Lee, Y.-W., Yun, S.-H., and Turgeon, B. G. 2003. Shifting fungal reproductive mode by manipulation of mating type genes: Obligatory heterothallism of Gibberella zeae. Mol. Microbiol. 50:145-152.

Lee, S., Son, H., Lee, J., Min, K., Choi, G. J., Kim, J.-C., and Lee, Y.-W. 2011. Functional analyses of two acetyl coenzyme A synthetases in the ascomycete Gibberella zeae. Eukaryot. Cell 10:1043-1052.

Lee, S.-H., Han, Y.-K., Yun, S.-H., and Lee, Y.-W. 2009a. Roles of the glyoxylate and methylcitrate cycles in sexual development and virulence in the cereal pathogen Gibberella zeae. Eukaryot. Cell 8:1155-1164.

Lee, S.-H., Lee, J., Lee, S., Park, E.-H., Kim, K.-W., Kim, M.-D., Yun, S.H., and Lee, Y.-W. 2009b. GzSNF1 is required for normal sexual and asexual development in the ascomycete Gibberella zeae. Eukaryot. Cell 8:116-127.

Leslie, J. F., and Summerell, B. A. 2006. The Fusarium Laboratory Manual. Blackwell Publishing, Ames, IA, U.S.A.

Li, Y., Wang, C., Liu, W., Wang, G., Kang, Z., Kistler, H. C., and Xu, J.-R. 2011. The $H D F 1$ histone deacetylase gene is important for conidiation, sexual reproduction, and pathogenesis in Fusarium graminearum. Mol. Plant-Microbe Interact. 24:487-496.

Lin, Y., Son, H., Lee, J., Min, K., Choi, G. J., Kim, J.-C., and Lee, Y.-W. 2011. A putative transcription factor MYT1 is required for female fertility in the Ascomycete Gibberella zeae. PLoS One 6:e25586.

Lin, Y., Son, H., Min, K., Lee, J., Choi, G. J., Kim, J.-C., and Lee, Y.-W. 2012. A putative transcription factor MYT2 regulates perithecium size in the ascomycete Gibberella zeae. PLoS One 7:e37859.

Livak, K. J., and Schmittgen, T. D. 2001. Analysis of relative gene expression data using real-time quantitative PCR and the $2^{-\triangle \mathrm{CT}}$ method. Methods 25:402-408.

Lu, S., Lyngholm, L., Yang, G., Bronson, C., Yoder, O., and Turgeon, B. G. 1994. Tagged mutations at the Tox1 locus of Cochliobolus heterostrophus by restriction enzyme-mediated integration. Proc. Natl. Acad. Sci. U.S.A. 91:12649-12653.

Maier, F. J., Miedaner, T., Hadeler, B., Felk, A., Salomon, S., Lemmens, M., Kassner, H., and Schaefer, W. 2006. Involvement of trichothecenes in fusarioses of wheat, barley and maize evaluated by gene disruption of the trichodiene synthase (Tri5) gene in three field isolates of different chemotype and virulence. Mol. Plant Pathol. 7:449-461.

Min, K., Son, H., Lim, J. Y., Choi, G. J., Kim, J.-C., Harris, S. D., and Lee, Y.-W. 2014. Transcription factor RFX1 is crucial for maintenance of genome integrity in Fusarium graminearum. Eukaryot. Cell 13:427436 .

Namiki, F., Matsunaga, M., Okuda, M., Inoue, I., Nishi, K., Fujita, Y., and Tsuge, T. 2001. Mutation of an arginine biosynthesis gene causes reduced pathogenicity in Fusarium oxysporum f. sp. melonis. Mol. PlantMicrobe Interact. 14:580-584.

Nathues, E., Joshi, S., Tenberge, K. B., von den Driesch, M., Oeser, B., Bäumer, N., Mihlan, M., and Tudzynski, P. 2004. CPTF1, a CREB-like transcription factor, is involved in the oxidative stress response in the phytopathogen Claviceps purpurea and modulates ROS level in its host Secale cereale. Mol. Plant-Microbe Interact. 17:383-393.

Nelissen, H., Fleury, D., Bruno, L., Robles, P., De Veylder, L., Traas, J., Micol, J. L., Van Montagu, M., Inzé, D., and Van Lijsebettens, M. 2005. The elongata mutants identify a functional Elongator complex in plants with a role in cell proliferation during organ growth. Proc. Natl. Acad. Sci. U.S.A. 102:7754-7759.
Nelissen, H., De Groeve, S., Fleury, D., Neyt, P., Bruno, L., Bitonti, M. B Vandenbussche, F., Van Der Straeten, D., Yamaguchi, T., and Tsukaya, H. 2010. Plant Elongator regulates auxin-related genes during RNA polymerase II transcription elongation. Proc. Natl. Acad. Sci. U.S.A. 107:1678-1683.

Otero, G., Fellows, J., Li, Y., de Bizemont, T., Dirac, A. M., Gustafsson, C. M., Erdjument-Bromage, H., Tempst, P., and Svejstrup, J. Q. 1999. Elongator, a multisubunit component of a novel RNA polymerase II holoenzyme for transcriptional elongation. Mol. Cell 3:109-118.

Proctor, R. H., Hohn, T. M., and McCormick, S. P. 1995. Reduced virulence of Gibberella zeae caused by disruption of a trichothecene toxin biosynthetic gene. Mol. Plant-Microbe Interact. 8:593-601.

Qi, W., Kwon, C., and Trail, F. 2006. Microarray analysis of transcript accumulation during perithecium development in the filamentous fungus Gibberella zeae (anamorph Fusarium graminearum). Mol. Genet. Genomics 276:87-100.

Sambrook, J., and Russell, D. W. 2001. Molecular Cloning: A Laboratory Manual. Cold Spring Harbor Laboratory Press, Cold Spring Harbor, NY, U.S.A

Schiestl, R. H., and Petes, T. D. 1991. Integration of DNA fragments by illegitimate recombination in Saccharomyces cerevisiae. Proc. Natl. Acad. Sci. U.S.A. 88:7585-7589.

Schouten, A., Tenberge, K. B., Vermeer, J., Stewart, J., Wagemakers, L., Williamson, B., and Van Kan, J. A. 2002. Functional analysis of an extracellular catalase of Botrytis cinerea. Mol. Plant Pathol. 3:227-238.

Seo, J.-A., Kim, J.-C., Lee, D.-H., and Lee, Y.-W. 1996. Variation in 8 ketotrichothecenes and zearalenone production by Fusarium graminearum isolates from corn and barley in Korea. Mycopathologia 134:31-37

Seong, K.-Y., Hou, Z., Tracy, M., Kistler, H. C., and Xu, J.-R. 2005. Random insertional mutagenesis identifies genes associated with virulence in the wheat scab fungus Fusarium graminearum. Phytopathology 95:744-750.

Skamnioti, P., Henderson, C., Zhang, Z., Robinson, Z., and Gurr, S. J. 2007. A novel role for catalase B in the maintenance of fungal cell-wall integrity during host invasion in the rice blast fungus Magnaporthe grisea. Mol. Plant-Microbe Interact. 20:568-580.

Son, H., Lee, J., Park, A. R., and Lee, Y.-W. 2011. ATP citrate lyase is required for normal sexual and asexual development in Gibberella zeae. Fungal Genet. Biol. 48:408-417.

Son, H., Lee, J., and Lee, Y.-W. 2012. Mannitol induces the conversion of conidia to chlamydospore-like structures that confer enhanced tolerance to heat, drought, and UV in Gibberella zeae. Microbiol. Res. 167:608615.

Son, H., Kim, M.-G., Min, K., Seo, Y.-S., Lim, J. Y., Choi, G. J., Kim, J.C., Chae, S.-K., and Lee, Y.-W. 2013. AbaA regulates conidiogenesis in the ascomycete fungus Fusarium graminearum. PLoS One 8:e72915.

Son, H., Kim, M.-G., Min, K., Lim, J. Y., Choi, G. J., Kim, J.-C., Chae, S.K., and Lee, Y.-W. 2014. WetA is required for conidiogenesis and conidium maturation in the ascomycete fungus Fusarium graminearum. Eukaryot. Cell 13:87-98.

Struhl, K. 1998. Histone acetylation and transcriptional regulatory mechanisms. Genes Dev. 12:599-606.

Sweigard, J. A., Carroll, A. M., Farrall, L., Chumley, F. G., and Valent, B. 1998. Magnaporthe grisea pathogenicity genes obtained through insertional mutagenesis. Mol. Plant-Microbe Interact. 11:404-412.

Tanabe, S., Ishii-Minami, N., Saitoh, K.-I., Otake, Y., Kaku, H., Shibuya, N., Nishizawa, Y., and Minami, E. 2011. The role of catalase-peroxidase secreted by Magnaporthe oryzae during early infection of rice cells. Mol. Plant-Microbe Interact. 24:163-171.

Torres, M. A., Jones, J. D., and Dangl, J. L. 2006. Reactive oxygen species signaling in response to pathogens. Plant Physiol. 141:373-378.

Trail, F., Gaffoor, I., and Vogel, S. 2005. Ejection mechanics and trajectory of the ascospores of Gibberella zeae (anamorph Fusarium graminearum). Fungal Genet. Biol. 42:528-533.

Vetting, M. W., S de Carvalho, L. P., Yu, M., Hegde, S. S., Magnet, S., Roderick, S. L., and Blanchard, J. S. 2005. Structure and functions of the GNAT superfamily of acetyltransferases. Arch. Biochem. Biophys. 433:212-226.

Walker, J., Kwon, S. Y., Badenhorst, P., East, P., McNeill, H., and Svejstrup, J. Q. 2011. Role of Elongator subunit Elp3 in Drosophila melanogaster larval development and immunity. Genetics 187:10671075

Wang, Y., Liu, W., Hou, Z., Wang, C., Zhou, X., Jonkers, W., Ding, S., Kistler, H. C., and Xu, J.-R. 2011. A novel transcriptional factor important for pathogenesis and ascosporogenesis in Fusarium graminearum. Mol. Plant-Microbe Interact. 24:118-128.

Winkler, G. S., Kristjuhan, A., Erdjument-Bromage, H., Tempst, P., and Svejstrup, J. Q. 2002. Elongator is a histone H3 and H4 acetyltransferase important for normal histone acetylation levels in vivo. Proc. Natl. 
Acad. Sci. U.S.A. 99:3517-3522.

Wittschieben, B. Ø., Otero, G., de Bizemont, T., Fellows, J., ErdjumentBromage, H., Ohba, R., Li, Y., Allis, C. D., Tempst, P., and Svejstrup, J. Q. 1999. A novel histone acetyltransferase is an integral subunit of elongating RNA polymerase II holoenzyme. Mol. Cell 4:123-128.

Wittschieben, B. Ø., Fellows, J., Du, W., Stillman, D. J., and Svejstrup, J. Q. 2000. Overlapping roles for the histone acetyltransferase activities of SAGA and Elongator in vivo. EMBO J. 19:3060-3068.

Wojtaszek, P. A. 1997. Oxidative burst: An early plant response to pathogen infection. Biochem. J. 322:681-692.

Wolffe, A. P., and Pruss, D. 1996. Targeting chromatin disruption: Tran- scription regulators that acetylate histones. Cell 84:817-819.

Yu, J.-H., Hamari, Z., Han, K.-H., Seo, J.-A., Reyes-Dominguez, Y., and Scazzocchio, C. 2004. Double-joint PCR: A PCR-based molecular tool for gene manipulations in filamentous fungi. Fungal Genet. Biol. 41:973-981.

\section{AUTHOR-RECOMMENDED INTERNET RESOURCE}

Fusarium Comparative database:

www.broadinstitute.org/annotation/genome/fusarium_graminearum 